\title{
THE M.H.D. VERSION OF A THEOREM OF H. WEYL
}

\author{
CHARLES C. CONLEY AND JOEL A. SMOLLER
}

\begin{abstract}
In his discussion of shock waves in arbitrary fluids, H. Weyl proves a theorem concerning the behavior of the entropy function along the Hugoniot curve. The analogous result is proven for the M.H.D. case.
\end{abstract}

The theorem of the title concerns the behavior of a function on a curve: The function represents entropy, the curve is the Hugoniot curve. Such a curve is determined for each point $\left(V_{0}, p_{0}\right)$ in the positive quadrant of the (specific) volume-pressure plane. Weyl [3] shows for gas dynamics that the entropy function restricted to this curve has at most one critical point, and that $\left(V_{0}, p_{0}\right)$ is the candidate. The significance of the result is that the entropy behaves in the physically expected way across shocks. For the general context the reader is referred to [2]; here we only want to point out how Weyl's clever argument works in the magnetohydrodynamic (m.h.d.) case.

The Hugoniot curve in the $(V, p)$ plane corresponding to the point $\left(V_{0}, p_{0}\right)$ is defined [1] by the equation

$$
H(V, p)=e-e_{0}+\frac{1}{2}\left(p+p_{0}\right)\left(V-V_{0}\right)+\phi(V)=0 .
$$

Here $e=e(V, p)$ represents internal energy and $e_{0}=e\left(V_{0}, p_{0}\right)$. The function $\phi(V)$ is identically zero in the case of gas dynamics while in the m.h.d. case $\phi$ takes the form

$$
\phi(V)=K\left(V-V_{0}\right)^{3}\left(V-V_{1}\right)^{-2}
$$

where $K$ and $V_{1}\left(\neq V_{0}\right)$ are positive constants (see [1]).

We use the following facts and hypothesis:

$$
\begin{aligned}
& \text { de }=-p d V+T d s, \text { where } T \text { and } s \text { denote respectively the } \\
& \text { temperature and entropy. }
\end{aligned}
$$

Given $V$ and $s$, a unique value $p=p(V, s)$ is determined. For the function so defined, $\partial^{2} p / \partial V^{2}$ and $\partial p / \partial s$ are both positive.

$$
\phi\left(V_{0}\right)=\phi^{\prime}\left(V_{0}\right)=0 \text { and } \phi^{\prime \prime}(V)\left(V-V_{0}\right) \geqq 0 \text {. }
$$

Received by the editors April 3, 1973.

AMS (MOS) subject classifications (1970). Primary 76L05, 76W05. 
For the $\phi$ of m.h.d. this is satisfied. In particular we compute

$$
\begin{aligned}
\phi^{\prime}(V)= & 3 K\left(V-V_{0}\right)^{2}\left(V-V_{1}\right)^{-2}-2 K\left(V-V_{0}\right)^{3}\left(V-V_{1}\right)^{-3}, \\
\phi^{\prime \prime}(V)= & 6 K\left(V-V_{0}\right)\left(V-V_{1}\right)^{-2}-12 K\left(V-V_{0}\right)^{2}\left(V-V_{1}\right)^{-3} \\
& +6 K\left(V-V_{0}\right)^{3}\left(V-V_{1}\right)^{-4}, \\
\phi^{\prime \prime}(V)\left(V-V_{0}\right)= & 6 K\left\{\left(V-V_{0}\right)\left(V-V_{1}\right)^{-1}-\left(V-V_{0}\right)^{2}\left(V-V_{1}\right)^{-2}\right\}^{2} \\
\geqq & 0 .
\end{aligned}
$$

The main lemma (8) used to examine the behavior of $s$ on the set where $H=0$ concerns the behavior of $s$ on a different family of curves. These are defined to be those curves restricted to which the one-form $\omega=d H-$ $T d s$ vanishes.

From (1), using (3), we compute

$$
d H=\left[\frac{1}{2}\left(p_{0}-p\right)+\phi^{\prime}(V)\right] d V+\frac{1}{2}\left[V-V_{0}\right] d p+T d s .
$$

Thus the integral curves of $\omega=0$ are solutions of the ordinary differential equation

$$
\dot{V}=V-V_{0}, \quad \dot{p}=p-p_{0}-2 \phi^{\prime}(V) .
$$

Using $\phi^{\prime}\left(V_{0}\right)=0$ in (5), observe that the point $\left(V_{0}, p_{0}\right)$ is a repelling rest point of these equations; we let $R$ denote the set of points in the positive $(V, p)$ quadrant which are in the domain of repulsion.

Note that if $\phi \equiv 0$ then $R$ is the whole positive quadrant. Also with the $\phi$ of m.h.d., $R$ contains precisely those points of the quadrant which lie on the same side of the line $V=V_{1}$ as does $V_{0}$. Namely, given any such point $(V, p)$, the $V$ component of the solution goes to $V_{0}$ as $t \rightarrow-\infty$. This, with $\phi^{\prime}\left(V_{0}\right)=0$, implies the $p$ component goes to $p_{0}$. Also, as the $V$ component tends to $V_{1}$, the absolute value of the $p$ component must go to infinity.

Now let $\gamma$ be any integral curve of (6) in $R$. The relevant facts about the behavior of $s$ on $\gamma$ are the following:

The critical points of $H \mid \gamma$ and $s \mid \gamma$ coincide.

This follows from $d H=\omega+T d s$.

(8) Any critical point of $s \mid \gamma$ is a maximum; in particular, $s \mid \gamma$ has at most one critical point.

To see this we observe there are two ways of writing the derivative, $\dot{p}$, of $p$ on $\gamma$; namely,

$$
p-p_{0}-2 \phi^{\prime}(V)=\dot{p}=p_{V} \dot{V}+p_{s} \dot{s} .
$$


Now differentiate this equation again along $\gamma$ and evaluate at a critical point of $s(\dot{s}=0)$. Writing 0 for terms involving $\dot{s}$ we have

$$
p_{V} \dot{V}+0-2 \phi^{\prime \prime}(V) \dot{V}=p_{V V} \dot{V}^{2}+0+p_{V} \ddot{V}+0+p_{s} \bar{s} .
$$

From (6) we see that $\ddot{V}=\dot{V}=V-V_{0}$ and so we conclude that

$$
\vec{s}=-p_{s}^{-1}\left[2 \phi^{\prime \prime}\left(V-V_{0}\right)+p_{V V}\left(V-V_{0}\right)^{2}\right] .
$$

Now from (4) and (5), $\tilde{s}<0$ so (8) is proved.

Weyl's statement now takes the form:

(9) THEOREM. If $(V, p) \neq\left(V_{0}, p_{0}\right)$ is a point in $R$ at which $H=0$ and $d H \neq 0$, then $\left.s\right|_{H=0}$ is not critical at $(V, p)$.

Proof. Since $d H \neq 0$ at $(V, p)$, the set $\{H=0\}$ meets a neighborhood of $(V, p)$ in a curve. Suppose the restriction of $s$ to this curve is critical at $(V, p)$; then, since $d H=\omega+T d s$, the restriction of $s$ to the integral curve of $(6)$ through $(V, p)$ is also critical at $(V, p)$. Consider the negative half orbit, say $\gamma$, from $\left(V_{0}, p_{0}\right)$ to $(V, p)$ (definition of $\left.R\right): H$ is zero at both ends of $\gamma$ so $H \mid \gamma$ is critical at some point of $\gamma$ strictly between $\left(V_{0}, p_{0}\right)$ and $(V, p)$. Since $H$ and $s$ are critical together on $\gamma$ in (7) we find that $s$ has two critical points on an orbit of (6). This contradicts (8).

In order not to spoil the proof, we have not investigated the question of existence of points in $\{H=0\}$ at which $d H=0$. In the case of a perfect gas, this does not happen.

\section{REFERENCES}

1. H. Cabannes, Theoretical magnetohydrodynamics, Academic Press, New York, 1970.

2. R. Courant and K. O. Friedrichs, Supersonic flow and shock waves, Interscience, New York, 1948. MR 10, 637.

3. H. Weyl, Shock waves in arbitrary fluids, Comm. Pure Appl. Math. 2 (1949), 103-122. MR 11, 626.

Department of Mathematics, University of Wisconsin, Madison, Wisconsin 53706 (Current address of C. C. Conley)

Current address (J. A. Smoller): Department of Mathematics, University of Michigan, Ann Arbor, Michigan 48104 